\title{
LE CHAMP LEXICAL DES PARTIES DU CORPS DANS L'ARGOT COMMUN DES JEUNES HONGROIS
}

\author{
Máté KovÁCS \\ Université Eötvös Loránd de Budapest
}

\begin{abstract}
En): This paper concerns the analysis of the lexical field of body parts in Hungarian youth slang. Dávid Szabó's doctoral thesis entitled L'argot des étudiants budapestois (The youth slang of Budapest), published in 2004, which contains in appendix a Hungarian-French bilingual dictionary of youth slang was taken as a point of departure for our own research. On the basis of Szabó's research and dictionary, our online questionnaire study had two main objectives: 1) to verify the frequency of use of words related to the lexical field of five body parts (mouth, legs, bottom, female and male genital organs) present in Szabó's dictionary and 2) to collect a new corpus composed of words presenting the actual usage. Our article concludes that Hungarian youth slang changes and does not change at the same time. More than twenty years after Szabó's research new words are of course used by young Hungarians but the various mechanisms governing word formation in Hungarian youth slang remain the same.
\end{abstract}

Keywords (En): body parts; lexical field; slang; Hungarian youth

Mots-clés (Fr) : parties du corps ; champ lexical ; argot commun ; jeunes Hongrois

DOI : $10.32725 /$ eer.2021.004

\section{Introduction}

Dans notre communication prononcée en 2019 lors du XIII ${ }^{e}$ Colloque international d'Argotologie à Paris, nous nous sommes proposé d'examiner le champ lexical d'aimer dans l'argot commun des jeunes Hongrois ${ }^{1}$. Le présent article constitue la suite de nos travaux fondés sur la thèse de Dávid Szabó, intitulée L'argot des étudiants budapestois, soutenue en 2002 sous la direction du professeur Jean-Pierre Goudaillier à l'Université René Descartes - Paris 5. Dans cette thèse, publiée deux ans plus tard sous forme remaniée aux éditions L'Harmattan (SZABÓ, 2004), l'auteur analyse l'argot des étudiants budapestois sur la base d'un corpus d'argot commun ${ }^{2}$ composé approximativement de 2300 mots et expressions recueillis dans le cadre d'une enquête conduite par l'auteur et ses étudiants au printemps 2000. La recherche visait une population de jeunes Budapestois âgés de 18 à 35 ans. Ce corpus, présenté en annexe de l'ouvrage sous forme d'un dictionnaire bilingue hongrois-français, nous servira, une fois de plus, de point de départ étant donné qu'il rassemble un grand nombre de mots relevant du champ lexical des parties du corps et utilisés dans l'argot commun hongrois.

\footnotetext{
${ }^{1}$ Voir Kovács (2021).

${ }^{2}$ Quant aux questions concernant les recherches dans le domaine de l'argot commun hongrois, voir aussi KIs (1997).
} 
Plus de vingt ans après la recherche menée par Dávid Szabó, nous avons refait une partie de son enquête afin de pouvoir observer le changement de l'argot commun $^{3}$ hongrois au fil du temps. Dans cette perspective, les objectifs de notre recherche étaient doubles. D'abord, nous voulions vérifier, en ayant recours à un questionnaire en ligne, le degré d'utilisation de certains mots relevant du champ lexical des parties du corps et présents dans le corpus de Dávid Szabó. Puis, nous avions l'intention de rassembler un nouveau corpus de mots témoignant de l'usage actuel. Dans ce qui suit, nous décrirons d'abord le déroulement de notre enquête pour ensuite détailler les résultats et, enfin, faire part des conclusions qui en découlent.

\section{Enquête}

Avant de parler des résultats de l'enquête, il nous semble important de présenter le déroulement et la méthodologie de notre recherche.

L'enquête a été réalisée par un questionnaire en ligne $e^{4}$ entre le 21 et le 31 janvier 2021. Rédigé en langue hongroise, le questionnaire contenait dix questions dont voici la traduction en français.

Question 1 : À quel point utilises-tu les mots suivants dans un cadre amical (avec des amis et copains de ton âge) pour désigner la bouche?

Question 2: Quels autres mots emploies-tu dans un cadre amical (avec des amis et copains de ton âge) pour désigner la bouche?

Question 3 : À quel point utilises-tu les mots suivants dans un cadre amical (avec des amis et copains de ton âge) pour désigner les jambes ?

Question 4 : Quels autres mots emploies-tu dans un cadre amical (avec des amis et copains de ton âge) pour désigner les jambes ?

Question $5:$ À quel point utilises-tu les mots suivants dans un cadre amical (avec des amis et copains de ton âge) pour désigner le postérieur ?

Question 6: Quels autres mots emploies-tu dans un cadre amical (avec des amis et copains de ton âge) pour désigner le postérieur?

Question 7 : À quel point utilises-tu les mots suivants dans un cadre amical (avec des amis et copains de ton âge) pour désigner les organes génitaux féminins?

Question 8: Quels autres mots emploies-tu dans un cadre amical (avec des amis et copains de ton âge) pour désigner les organes génitaux féminins ?

Question 9: À quel point utilises-tu les mots suivants dans un cadre amical (avec des amis et copains de ton âge) pour désigner les organes génitaux masculins ?

Question 10: Quels autres mots emploies-tu dans un cadre amical (avec des amis et copains de ton âge) pour désigner les organes génitaux masculins?

Comme il ressort des questions ci-dessus, nous avons choisi cinq parties du corps (la bouche, les jambes, le postérieur, les organes génitaux féminins et masculins) dont nous avons soumis les dénominations à l'analyse. Ces questions s'articulent autour de nos deux objectifs de recherche. Dans le cas des questions 1,

\footnotetext{
${ }^{3}$ Nous définissons l'argot commun comme un type d'argot « qui est pratiqué, indépendamment de toute appartenance à un groupe social, par une large fraction de la population » (FRANÇOIS, $1975: 6$ ). ${ }^{4}$ Le questionnaire a été conçu et partagé en ligne via Google Drive.
} 
3, 5, 7 et 9, nous voulions mesurer le degré d'utilisation des mots présents dans le corpus de Dávid Szabó. Les participants étaient ainsi amenés à évaluer, sur un barème de cinq points, la fréquence d'usage de douze mots sélectionnés pour chaque partie du corps. À l'aide des questions $2,4,6,8$ et 10 nous avons pu rassembler un nouveau corpus mettant en scène l'usage actuel.

Notre questionnaire a été renseigné au total par 120 participants âgés de 18 à 35 ans ${ }^{5}$, dont une large majorité de femmes : 93 femmes (77,5\%) contre 27 hommes $(22,5 \%)$. Parmi ces 120 participants, $81 \%$ sont élèves ou étudiant(e)s, alors que $19 \%$ sont des employé(e)s. Afin de nuancer l'analyse, nous avons divisé la tranche d'âge retenue en deux sous-tranches : les participants âgés de 18 à 24 ans (69\%) dominent l'échantillon et ceux âgés de 25 à 35 ans en constituent $31 \%$. À part le sexe, l'âge et la profession, nous avons également pris en compte comme variable sociale $^{6}$ le lieu de résidence. $63 \%$ des participants de notre enquête habitent la capitale, Budapest, $9 \%$ résident dans un chef-lieu de département, $23 \%$ dans une ville et $5 \%$ dans une commune.

\section{Résultats de l'enquête}

Dans la suite, nous ferons part des résultats de notre recherche. Pour les cinq parties du corps sélectionnées (la bouche, les jambes, le postérieur, les organes génitaux féminins et masculins), nous examinerons d'abord la fréquence d'utilisation de douze dénominations choisies dans le corpus de Dávid Szabó, puis nous présenterons les «nouveaux » mots recueillis dans le cadre de notre enquête.

\subsection{L'usage des mots désignant la bouche relevés dans le corpus de Dávid Szabó}

La première partie du corps dont les diverses dénominations font l'objet d'analyse est la bouche. Sur la base des résultats obtenus pour notre questionnaire, nous avons classé les mots dans trois catégories comme le tableau ci-dessous en rend compte.

\begin{tabular}{|l|}
\hline Mots fréquemment utilisés $(\mathbf{0}$ mot $\mathbf{- 0} \%)$ \\
\hline Mots rarement utilisés (1 mot $\mathbf{- 8} \%)$ \\
csőr « bec » \\
\hline Mots nullement utilisés (11 mots - $\mathbf{9 2} \%)$ \\
bagóleső « qui guette la chique », csicsergő « gazouillant », csipogó « pépiant », \\
csócsáló « mastiquant », etető « mangeoire », fecsegő « bavardant », nyalakodó « qui \\
goûte », pampogó « qui rouspète », pampula (< slov. papula « gueule »), pofanyílás \\
« ouverture de la gueule », tátogó « qui s’ouvre » \\
\hline
\end{tabular}

Tableau 1 - Degré d'utilisation des mots du corpus pour dénommer la bouche

\footnotetext{
${ }^{5}$ Dans cette enquête, nous avons opté pour les mêmes tranches d'âge que Dávid Szabó pour assurer une bonne comparabilité des résultats.

${ }^{6}$ Sur les variables, voir par exemple CALVET (2011).
} 
Les participants de notre enquête déclarent n'utiliser presque aucun mot que nous avons sélectionné dans le corpus de Dávid Szabó. Il n’y a qu'un seul élément : csör $(8 \%)$ qui est rarement employé par les participants. Ceci montre que les locuteurs semblent avoir recours à l'usage d'autres mots qui seront abordés par la suite.

\subsection{D'autres mots actuellement utilisés pour désigner la bouche}

À part les douze mots utilisés de manière sporadique, les participants de l'enquête en ont partagé avec nous d'autres employés pour renvoyer à la bouche.

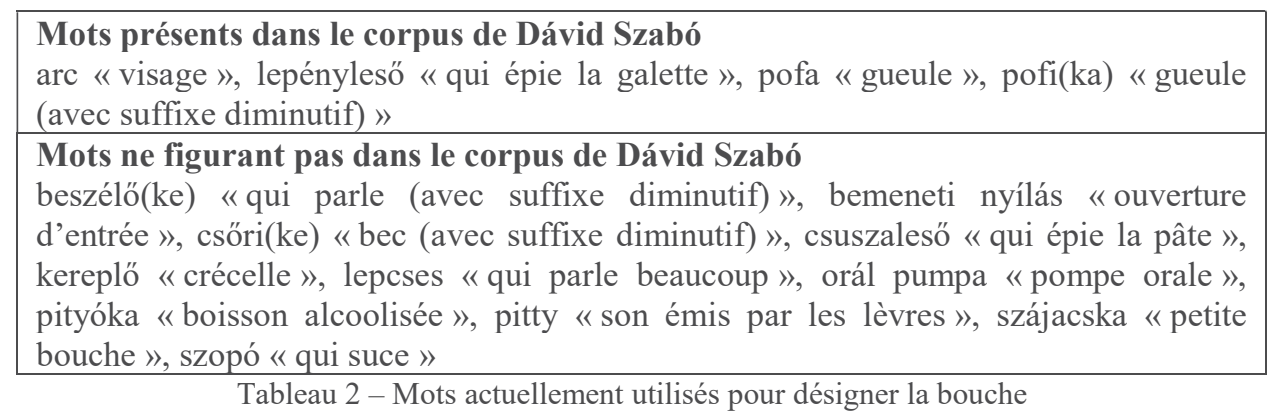

Comme le tableau ci-dessus en témoigne, certains mots se trouvent également

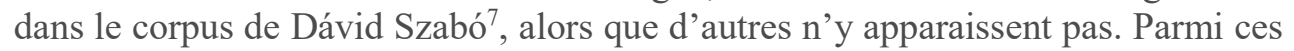
mots, divers procédés de formation lexicale peuvent être observés (SZABÓ 2002a, 2004). Des procédés formels tels que la suffixation se manifeste dans le cas de beszélö $+k e$ et csöri+ke, et la composition se présente, par exemple, dans csuszalesö $<$ csusza «pâte» + lesö « qui épie» < les « épie » + suffixe participial -ö, littéralement "qui épie la pâte ». D'autres mots mettent en scène des procédés sémantiques comme la métaphore : bemeneti nyílás « ouverture d'entrée », kereplö « crécelle », orál pumpa « pompe orale », etc.

\subsection{L'usage des mots désignant les jambes relevés dans le corpus de Dávid Szabó}

D'après les résultats de notre enquête, la fréquence d'utilisation des dénominations des jambes montre un aspect légèrement différent par rapport à l'exemple précédent.

\footnotetext{
${ }^{7}$ Rappelons que nous n'avons choisi que douze mots pour chaque partie du corps dans le corpus de Dávid Szabó.
} 


\begin{tabular}{|c|}
\hline Mots fréquemment utilisés ( 0 mots - 0 \%) \\
\hline $\begin{array}{l}\text { Mots rarement utilisés (3 mots }-25 \% \text { ) } \\
\text { csülök « jambonneau », pipaszár « tuyau d'une pipe », virgács « badine » }\end{array}$ \\
\hline $\begin{array}{l}\text { Mots nullement utilisés }(\mathbf{9} \text { mots }-\mathbf{7 5} \%) \\
\text { alváz « châssis (d'une automobile) », csaniga (< hongr. csaniga " jambe »), csánk } \\
(<\text { tsig. chang « jambe »), futómű « train de roulement », mankó « béquilles », oszlop } \\
\text { « colonne », pálca « baguette », szipka « fume-cigarette », venyige « sarment (de } \\
\text { vigne) » }\end{array}$ \\
\hline
\end{tabular}

Tableau 3 - Degré d'utilisation des mots du corpus pour dénommer les jambes

Comme le tableau 3 le montre, si trois sur douze mots, à savoir $25 \%$ des éléments, semblent rarement employés par les participants, la grande majorité (75\%) demeure non utilisée et est, par conséquent, remplacée par d'autres expressions. Mais avant d'analyser les mots recueillis dans le cadre de notre enquête, il nous semble intéressant de présenter l'emploi du mot csülök « jambonneau $»^{8}$ selon les différentes variables.

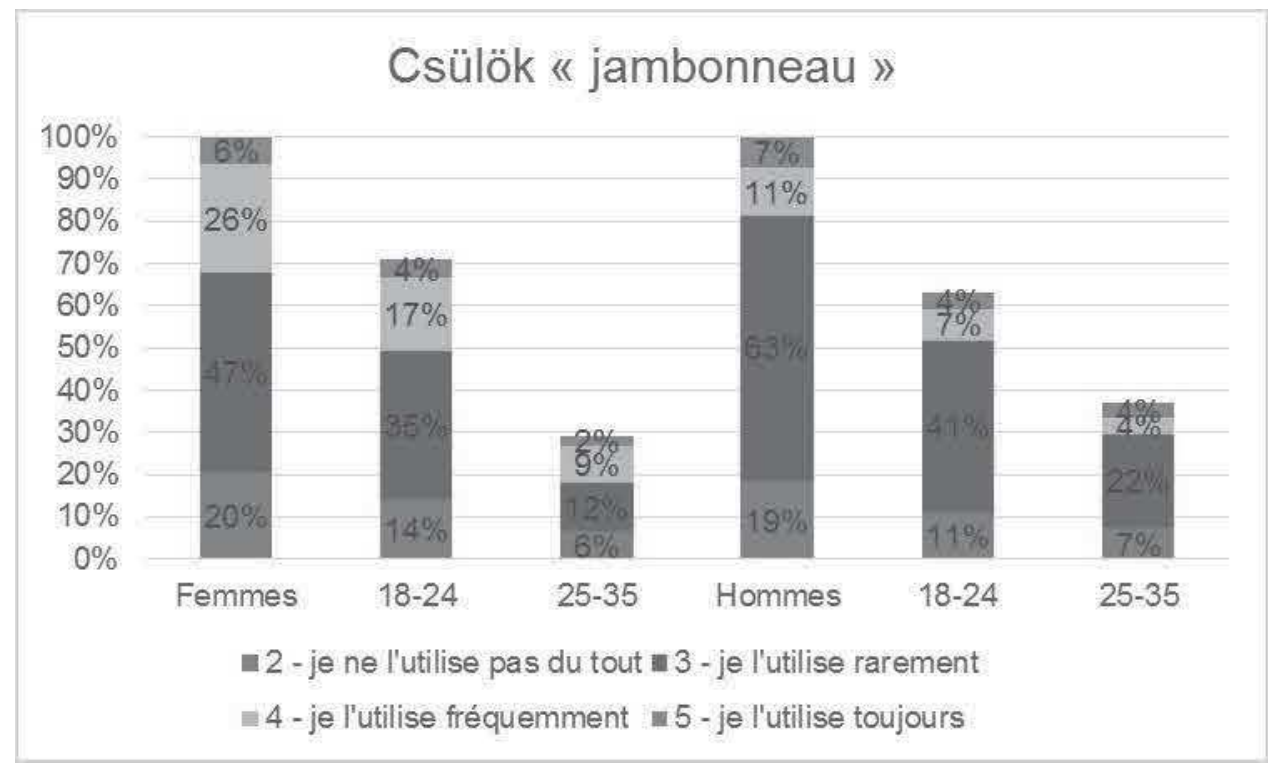

Figure 1 - L'utilisation du mot csülök selon le sexe et l'âge

D'après la figure 1, les femmes ayant participé à notre enquête déclarent utiliser ce mot plus fréquemment que les hommes : $32 \%$ pour des usages constant et fréquent pour les femmes contre $18 \%$ pour les hommes. Cette différence d'emploi semble se confirmer clairement dans la tranche d'âge de 18 à 24 ans avec $21 \%$ pour les utilisations constante et fréquente pour les femmes contre $11 \%$ pour les hommes et aussi, dans une moindre mesure, parmi les participants âgés de 25 à 35 ans avec $11 \%$ pour les femmes contre $8 \%$ pour les hommes.

À part la différence suivant le sexe, il est également possible de constater une divergence selon la profession et le lieu de résidence de nos participants.

\footnotetext{
${ }^{8}$ Faute d'espace, nous ne détaillerons que l'utilisation d'un seul mot pour chaque partie du corps.
} 


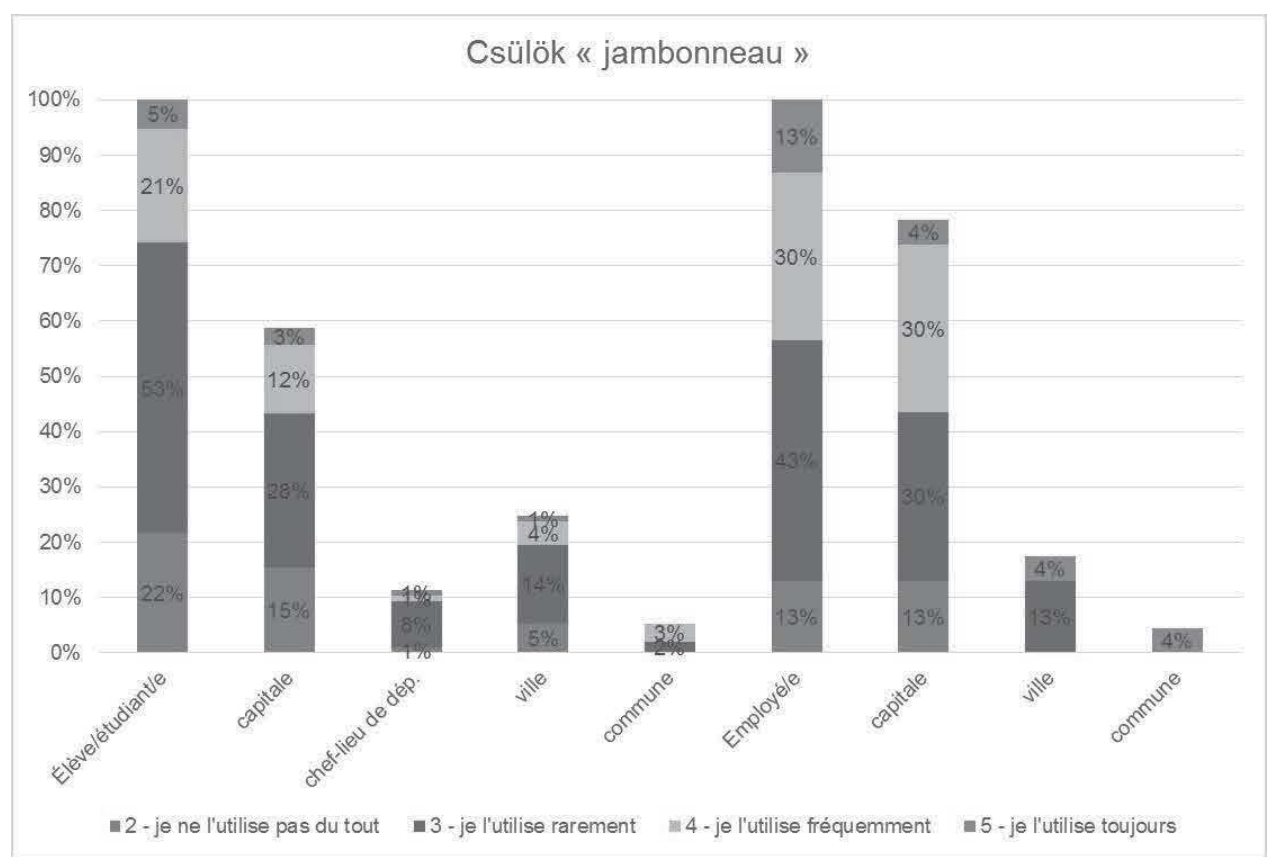

Figure 2-L'utilisation du mot csülök selon la profession et le lieu de résidence

Les résultats de notre enquête témoignent du fait que les employés (43\%) semblent se servir du mot csülök plus que les élèves et étudiants (26\%). De plus, ce mot est le plus fréquemment utilisé par les employés de la capitale avec $34 \%$ pour les usages constant et fréquent.

\subsection{D'autres mots actuellement utilisés pour désigner les jambes}

Pour la dénomination des jambes, les participants de notre enquête ne manquent pas de mots, comme le tableau suivant en témoigne.

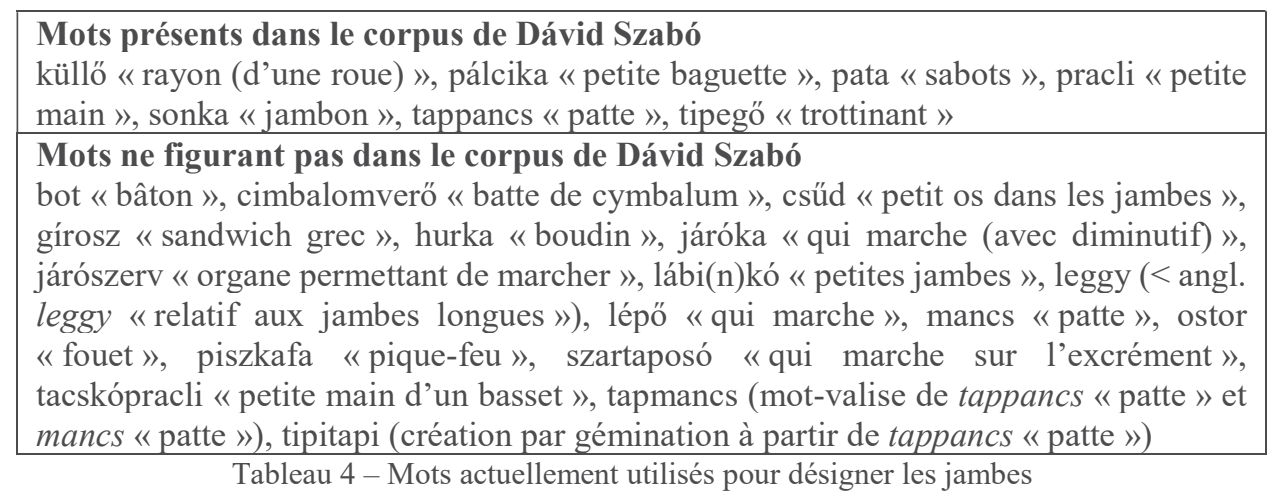

À l'instar du cas précédent, certains mots mentionnés par les participants sont inclus dans le dictionnaire bilingue de Dávid Szabó, mais un nombre plus important 
n'y est pas présent. Il nous semble important d'y remarquer divers processus de création lexicale (SZABÓ 2002a, 2004). Parmi les procédés formels, nous pouvons mentionner la composition : cimbalomverö < cimbalom « cymbalum $»^{9}+$ verö « qui bat »< ver « bat »+ suffixe participial -ö et járószerv < járó « qui marche »<jár « marche » + suffixe participial $-\dot{o}+$ szerv « organe »; le mot-valise : tapmancs < tappancs «patte»+ mancs "patte» ou la suffixation : járó $+k a$. Les processus sémantiques sont également présents avec, par exemple, la métaphore: bot «bâton», gírosz «sandwich grec», piszkafa «pique-feu», etc. Enfin, nous pouvons observer un exemple d'emprunt ${ }^{10}$ : leggy < angl. leggy « relatif aux jambes longues ${ }^{11}$. De manière générale, il convient d'ajouter que même si notre question se rapportait aux diverses désignations des jambes, certains mots évoqués par les participants (p. ex. mancs, pata, tacskópracli, tapmancs, tipitapi, etc.) semblent plutôt renvoyer aux pieds.

\subsection{L'usage des mots désignant le postérieur relevés dans le corpus de Dávid Szabó}

D'après notre enquête, les dénominations relatives au postérieur et présentes dans le corpus de Dávid Szabó montrent une tendance d'utilisation plus importante que celles faisant référence aux parties du corps évoquées précédemment.

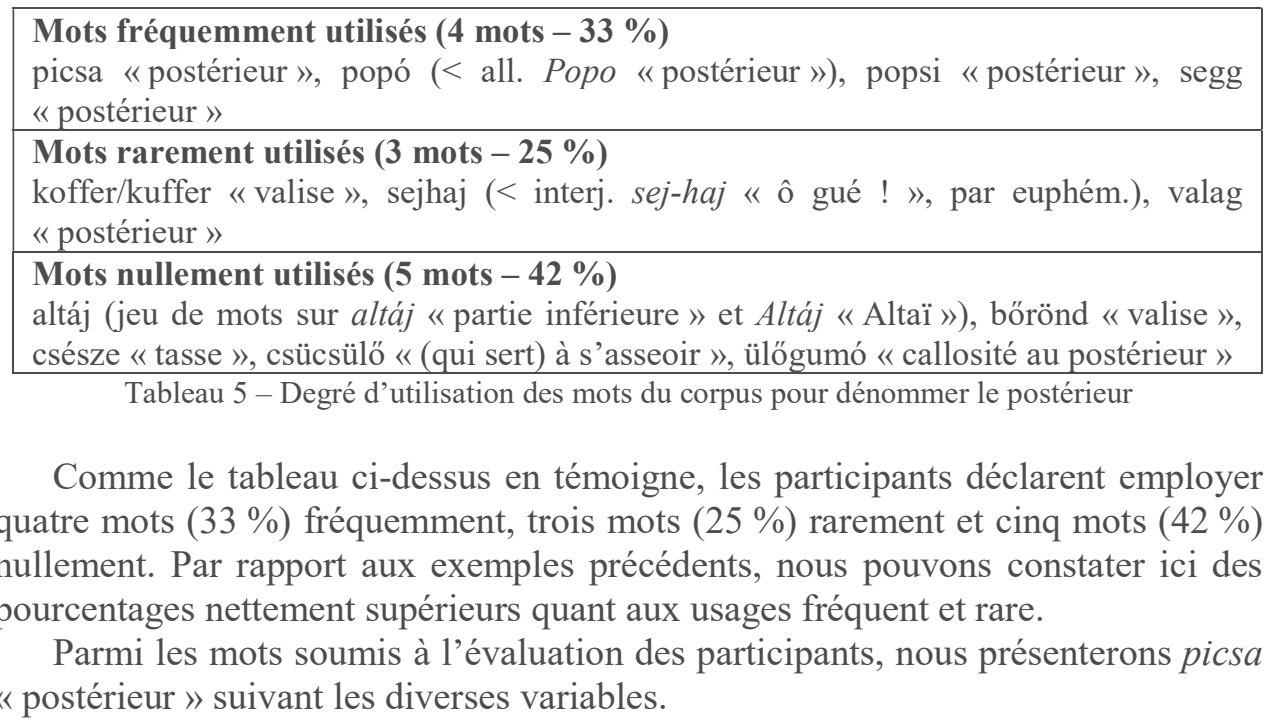

\footnotetext{
${ }^{9}$ Remarquons que le cymbalum est un instrument de musique à cordes frappées souvent utilisé dans la musique folklorique hongroise.

${ }^{10}$ Sur la place de l'emprunt dans l'argot commun hongrois, voir par exemple SzABÓ (2002b).

${ }^{11}$ Pour la précision, leggy est un adjectif en anglais mais il est utilisé ici comme un substantif : il s'agit donc d'un cas de conversion (dérivation impropre).
} 


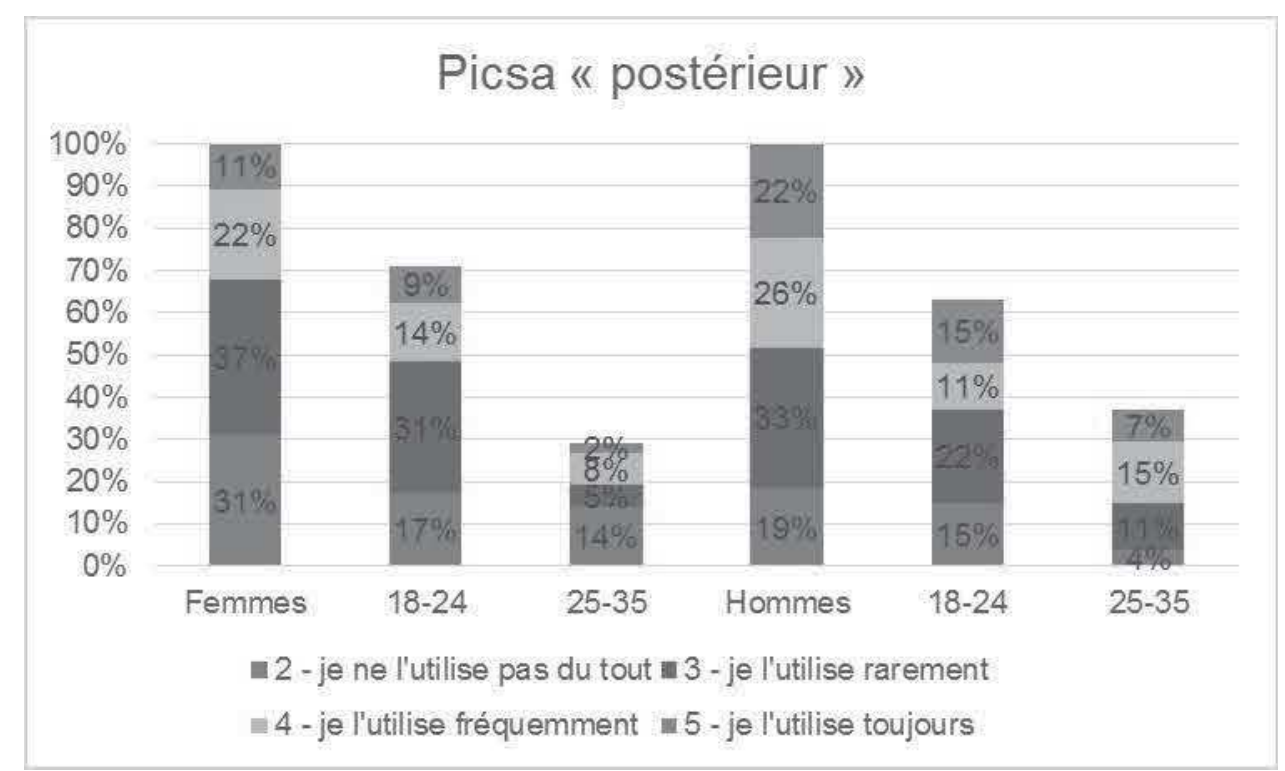

Figure 3 - L'utilisation du mot picsa selon le sexe et l'âge

Selon la figure 3, une différence importante peut être observée dans l'usage que font de ce mot les femmes et les hommes. Si $33 \%$ des femmes déclarent l'employer constamment ou fréquemment, pour ces mêmes usages le pourcentage des hommes s'élève à $48 \%$. Cette divergence se manifeste dans les deux tranches d'âge, plus particulièrement chez les participants âgés de 25 à 35 ans : $10 \%$ pour les femmes contre $22 \%$ des hommes.

La profession et le lieu de résidence comme variables semblent également témoigner d'une différence quant à l'emploi du mot picsa.

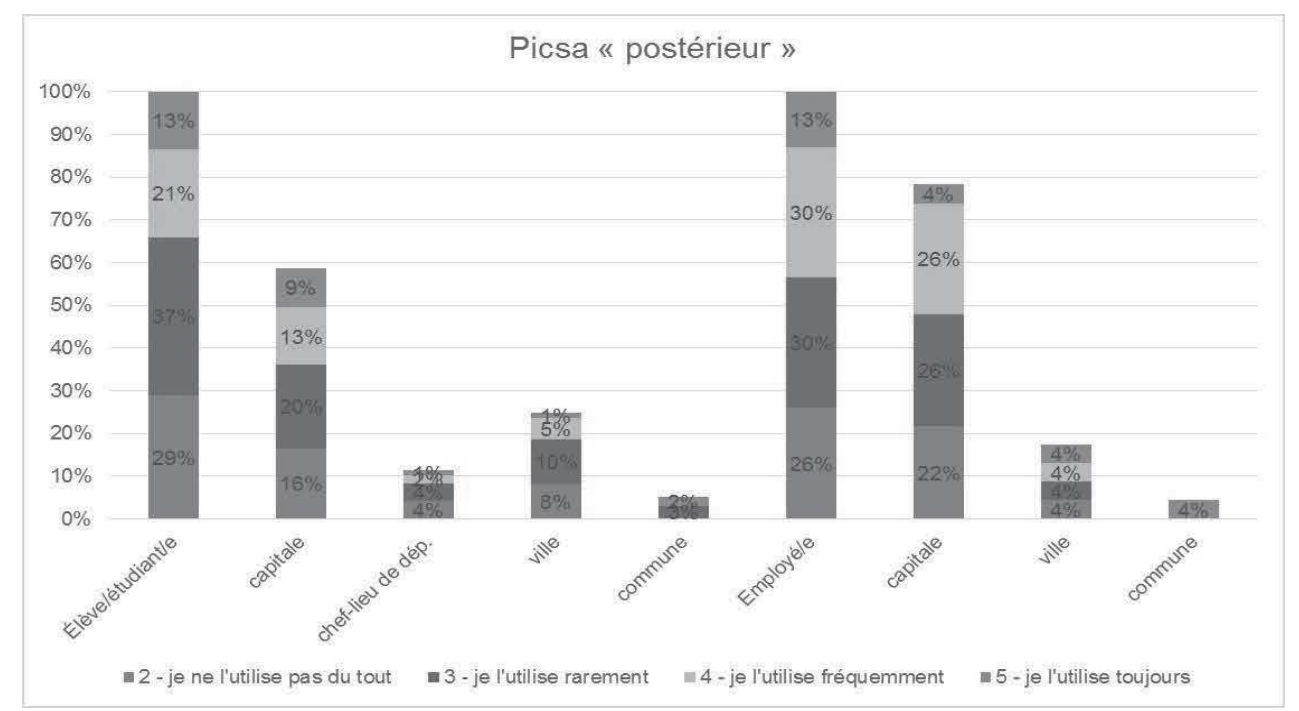

Figure 4 - L'utilisation du mot picsa selon la profession et le lieu de résidence 
Comme nous pouvons le constater sur la figure 4 , les employés déclarent utiliser ce mot plus fréquemment que les élèves et étudiants : $43 \%$ pour les employés contre $34 \%$ pour les élèves et étudiants pour les usages constant et fréquent. Cette même différence semble également se confirmer chez les habitants de la capitale, des villes et communes ${ }^{12}$.

\subsection{D'autres mots actuellement utilisés pour désigner le postérieur}

Le vocabulaire de nos participants semble comporter un grand nombre d'exemples pour parler du postérieur.

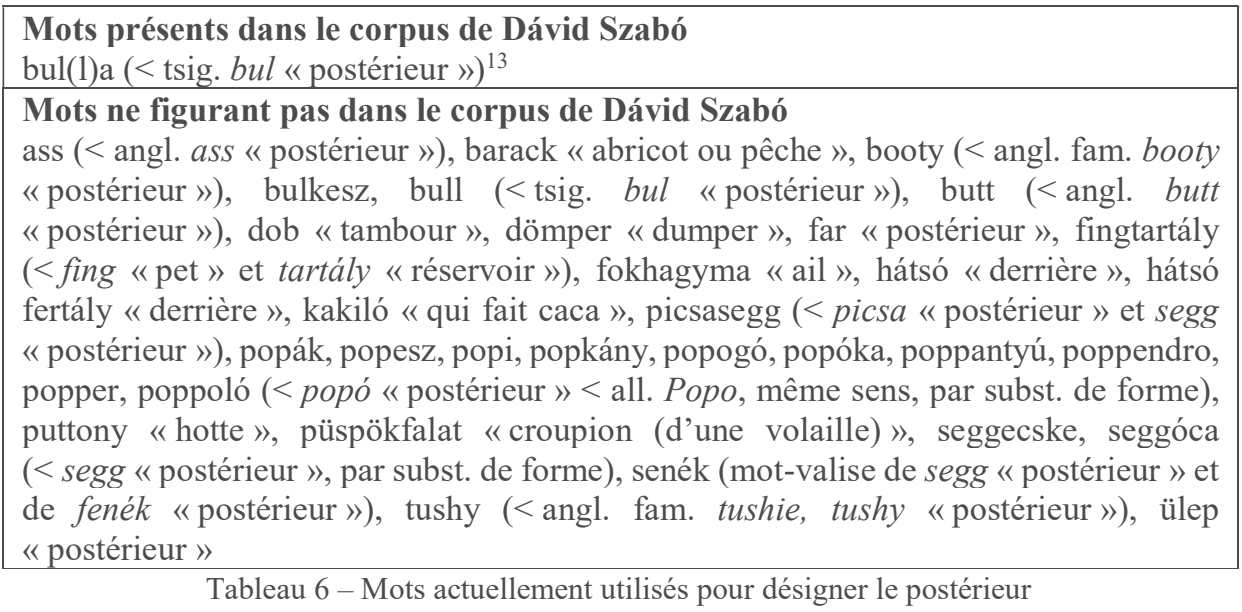

Comme le tableau ci-dessus l'illustre, presque aucun mot mentionné par les participants n'est présent dans le corpus de Dávid Szabó. Mais, au niveau des procédés de création lexicale (SZABÓ 2002a, 2004), nous retrouvons les mêmes mécanismes. En ce qui concerne l'aspect formel, il est important d'évoquer la suffixation: segg+ecske, segg+óca, la composition: picsasegg < picsa «postérieur »+segg «postérieur » et le mot-valise : senék < segg «postérieur »+ fenék «postérieur ». Les procédés sémantiques se manifestent surtout sous forme de métaphores : barack « abricot ou pêche » ${ }^{14}$, dob «tambour », fokhagyma « ail », etc., ces exemples étant utilisés pour dénommer le postérieur sur la base d'une relation de similarité, notamment d'une ressemblance de forme. Les emprunts, surtout à l'anglais mais également à d'autres langues (tsigane, allemande) $)^{15}$, y sont

12 Remarquons que dans notre échantillon nous n'avons pas d'employé habitant un chef-lieu de département.

${ }^{13}$ Attesté aussi dans le sens de « vulve » chez SzABÓ (2004: 258) et KIs (2008: 105).

${ }^{14}$ Il convient de remarquer que selon notre corpus, barack «abricot ou pêche» ne désigne pas seulement le postérieur mais également les organes génitaux féminins. Il s'agit dans les deux cas d'une métaphore basée sur un rapport de similitude, ce qui peut expliquer l'usage du même mot pour dénoter deux parties du corps.

${ }^{15}$ Précisons que les emprunts aux langues tsigane et allemande sont de vieux emprunts qui ne sont donc pas entrés récemment dans l'argot commun hongrois. 
nombreux, par exemple : ass < angl. ass « postérieur », booty < angl. fam. booty « postérieur », bulkesz, bull < tsig. bul « postérieur».

\subsection{L'usage des mots désignant les organes génitaux féminins relevés dans le corpus de Dávid Szabó}

Les diverses dénominations concernant les organes génitaux féminins sélectionnées dans le corpus de Dávid Szabó semblent être peu employées actuellement.

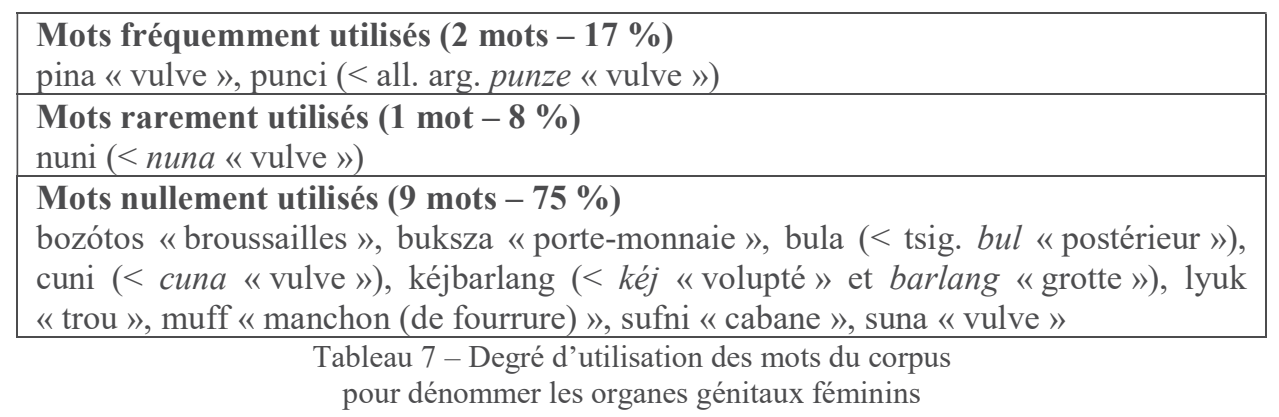

Comme notre tableau le montre, selon l'évaluation des participants seuls deux mots $(17 \%)$ sont utilisés fréquemment, un $(8 \%)$ rarement et neuf $(75 \%)$ nullement. Ces données statistiques ressemblent surtout à celles observées à propos de l'usage des désignations des jambes.

Parmi les mots inclus dans le tableau 7, nous avons choisi pina « vulve» pour l'analyse selon les variables.

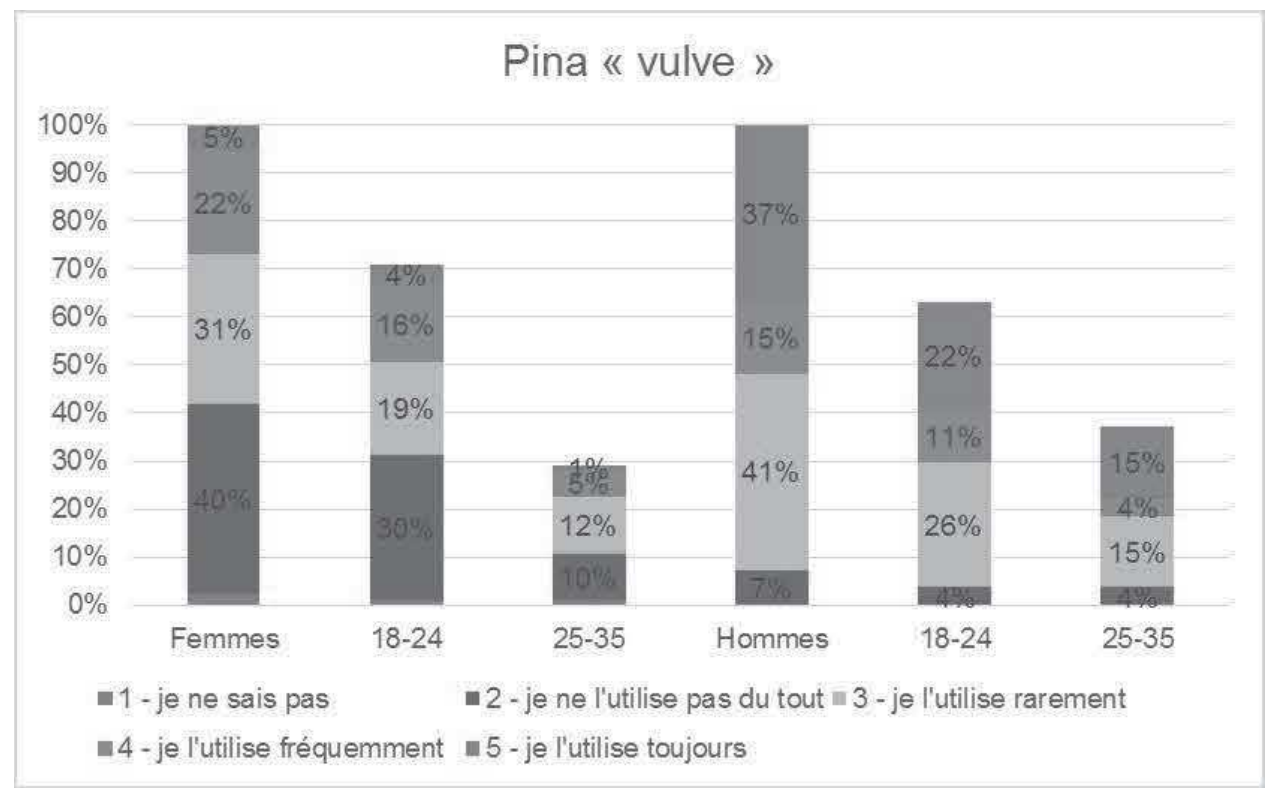

Figure 5 - L'utilisation du mot pina selon le sexe et l'âge 
Il convient d'observer que pina «vulve» semble être un mot d'homme, tout comme le précédent picsa « postérieur $»^{16} .52 \%$ des hommes participant à notre enquête déclarent l'employer de manière constante ou fréquente, alors que pour ces mêmes usages le pourcentage des femmes n'atteint que $27 \%$. Les deux tranches d'âge confirment clairement cette tendance : $33 \%$ pour les hommes contre $20 \%$ pour les femmes chez les participants âgés de 18 à 24 ans, $19 \%$ pour les hommes contre $6 \%$ pour les femmes chez ceux âgés de 25 à 35 ans.

La profession et le lieu de résidence présentent également une différence dans l'usage du mot pina.

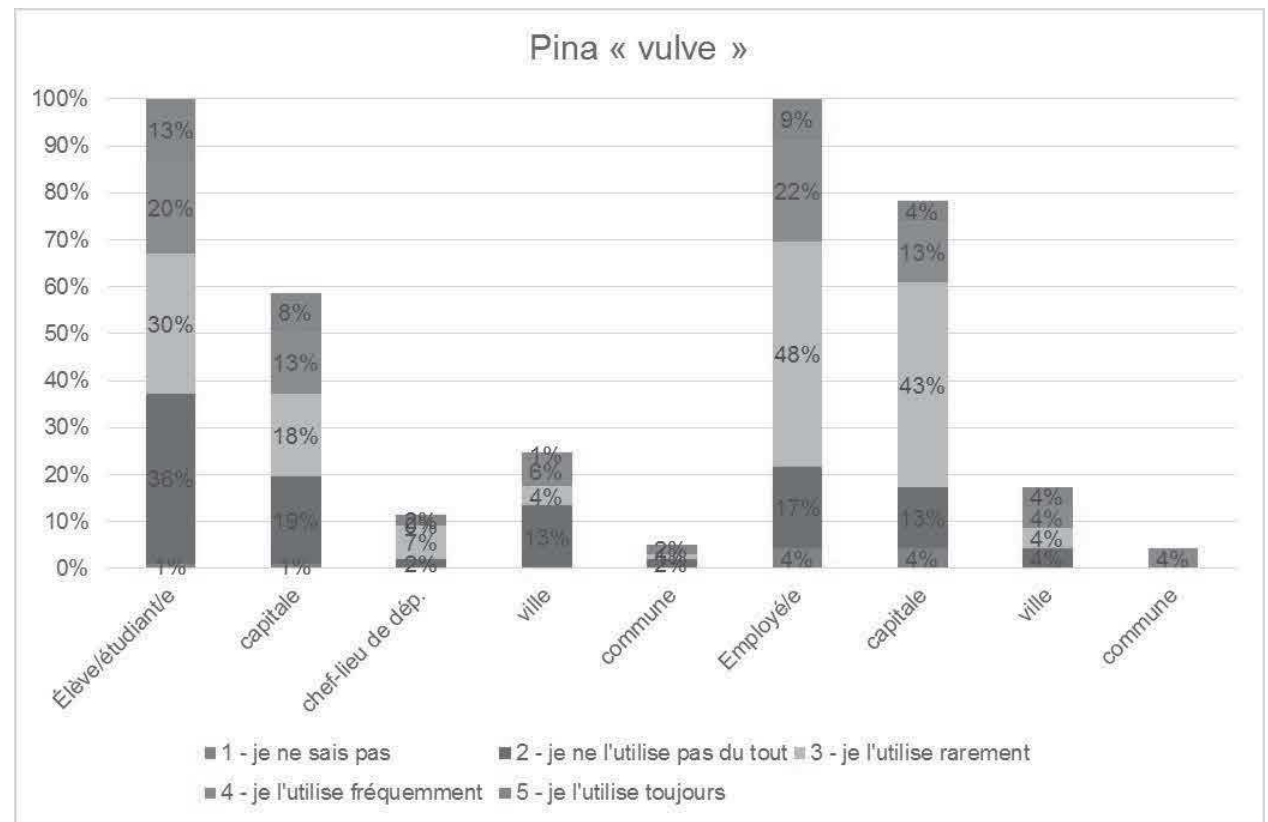

Figure 6 - L'utilisation du mot pina selon la profession et le lieu de résidence

De manière générale, les employés semblent utiliser ce mot plus fréquemment que les élèves et étudiants : $79 \%$ pour les employés pour les usages constant, fréquent et rare contre $63 \%$ pour les élèves et étudiants pour les mêmes utilisations. Cela se confirme plus spécifiquement chez les habitants de la capitale $(60 \%$ pour les employés contre $39 \%$ pour les élèves et étudiants), alors que la différence est peu significative chez ceux habitant les villes ou les communes.

\subsection{D'autres mots actuellement utilisés pour désigner les organes génitaux féminins}

D'après les résultats de notre enquête, les organes génitaux féminins possèdent une grande richesse de dénominations dans l'argot commun des jeunes. Le tableau ci-dessous présente les exemples relevés dans notre questionnaire.

16 À ce propos, voir SZABÓ (2009). 


\begin{abstract}
Mots présents dans le corpus de Dávid Szabó
mindzsó (< tsig., sinto minč « vulve »), nuna (< peut-être hgr. fam. puna « vulve »), nunci (< nuna « vulve»), picsa « vulve», puna « vulve », punca (< all. arg. punze « vulve »), vágás « coupure »

\section{Mots ne figurant pas dans le corpus de Dávid Szabó}

alváz « châssis (d'une automobile) », barack « abricot ou pêche », barlang " grotte », bibi(ke) « vulve (avec diminutif) », bun (< angl. bun « pain »), cuncimókus (< cunci « vulve » et mókus «écureuil »), csúnya «moche », garázs « garage », kagyló « coquillage », kisfazék «petite casserole», kispicsa «petite vulve », málnás « framboisier», mézesbödön (< mézes « relatif au miel» et bödön «pot»), mézescsupor $(<\text { mézes « relatif au miel» et csupor « pot») })^{17}$, nunetta 《 vulve », nunus «vulve», peach (< angl. peach «pêche»), pinácska «vulve (avec diminutif) », pinarelló « vulve », pinasen « vulve », pini « vulve », punetti « vulve », pun(sz)i « vulve », punus « vulve ${ }^{18}$, pussy (< angl. fam. pussy « vulve »), puszpusz (< angl. fam. pussy «vulve », par redoublement), repedés « fissure », rózsabimbó (<rózsa « rose » et bimbó « bouton »), saláta « salade », sun(c)i « vulve », suncica (mot-valise de sunci «vulve» et cica «chat»), sunner «vulve», sündisznó « hérisson », szaporodólyuk (< szaporodó « qui reproduit » et lyuk « trou »), szentély « sanctuaire », tevepata (< teve « chameau » et pata « patte »), vaginina (< vagina « vagin »), zsuzsi $(<$ suni, par attraction paronymique de Zsuzsi, diminutif du prénom Zsuzsanna « Suzanne »)
\end{abstract}

Tableau 8 - Mots actuellement utilisés pour désigner les organes génitaux féminins

Pareillement aux trois exemples évoqués précédemment, de nombreuses dénominations des organes génitaux féminins rapportées par nos participants ne sont pas présentes dans le corpus de Dávid Szabó. Quant aux processus de création lexicale (SZABÓ 2002a, 2004), nous retrouvons, une fois de plus, un certain nombre de procédés déjà observés et d'autres dont nous n'avons pas encore fait mention. Parmi les processus formels, la composition apparaît dans les exemples tels que rózsabimbó < rózsa « rose » + bimbó « bouton » ou szaporodólyuk < szaporodó

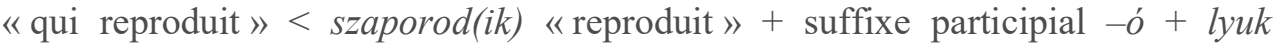
《 trou », etc., le mot-valise est représenté par suncica < sunci « vulve» + cica « chat » et la suffixation (précédée de troncation), par exemple, par nunus < nun(a) + suffixe -us. Deux autres procédés formels, non mentionnés pour l'instant, figurent aussi parmi nos exemples : la troncation suivie de redoublement (puszpusz < angl. fam. pussy 《vulve $\left.»^{19}\right)$ et l'attraction paronymique (zsuzsi $<$ suni, par attraction paronymique de Zsuzsi, diminutif du prénom Zsuzsanna «Suzanne »). En ce qui concerne les procédés sémantiques, ils se mettent en scène notamment sous forme de métaphore : barlang " grotte », garázs « garage », etc. Notre corpus comprend également plusieurs emprunts : mindzsó (< tsig., sinto minč «vulve»), peach (< angl. peach « pêche »), etc. Remarquons enfin que certains mots listés dans notre tableau figurent tout de même dans le dictionnaire de Dávid Szabó mais avec une

\footnotetext{
${ }^{17}$ Si mézesbödön et mézescsupor ne se trouvent pas dans le dictionnaire de Dávid Szabó, un autre synonyme mézesköcsög y est inclus (SZABÓ, 2004 : 295).

${ }^{18}$ Remarquons que des mots comme pinácska, pinarelló, pinasen, pini, punetti, pun(sz)i et punus sont des variantes de pina, ce dernier étant présent dans le corpus de Dávid Szabó.

${ }^{19}$ Cela peut être également un exemple pour l'attraction paronymique de puszipuszi < puszi « bisous ».
} 
ou plusieurs significations différentes. Tel est le cas, par exemple, des mots comme alváz « postérieur », « jambes » et kagyló « oreille».

\subsection{L'usage des mots désignant les organes génitaux masculins relevés dans le corpus de Dávid Szabó}

La dernière partie du corps dont les dénominations font l'objet d'analyse est les organes génitaux masculins.

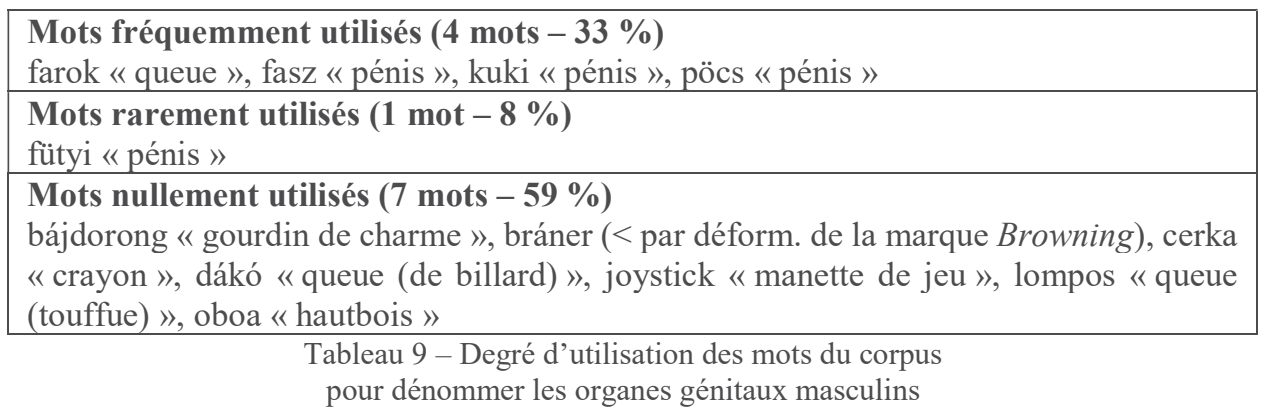

Parmi les mots choisis dans le corpus de Dávid Szabó, quatre (33\%) semblent fréquemment utilisés par les participants, un $(8 \%)$ rarement et sept $(59 \%)$ nullement. Néanmoins, comme nous le verrons par la suite, d'autres mots faisant partie du dictionnaire de Dávid Szabó demeurent à l'usage selon le témoignage de nos participants. Avant d'analyser ces expressions, regardons de plus près le mot fasz « pénis ».

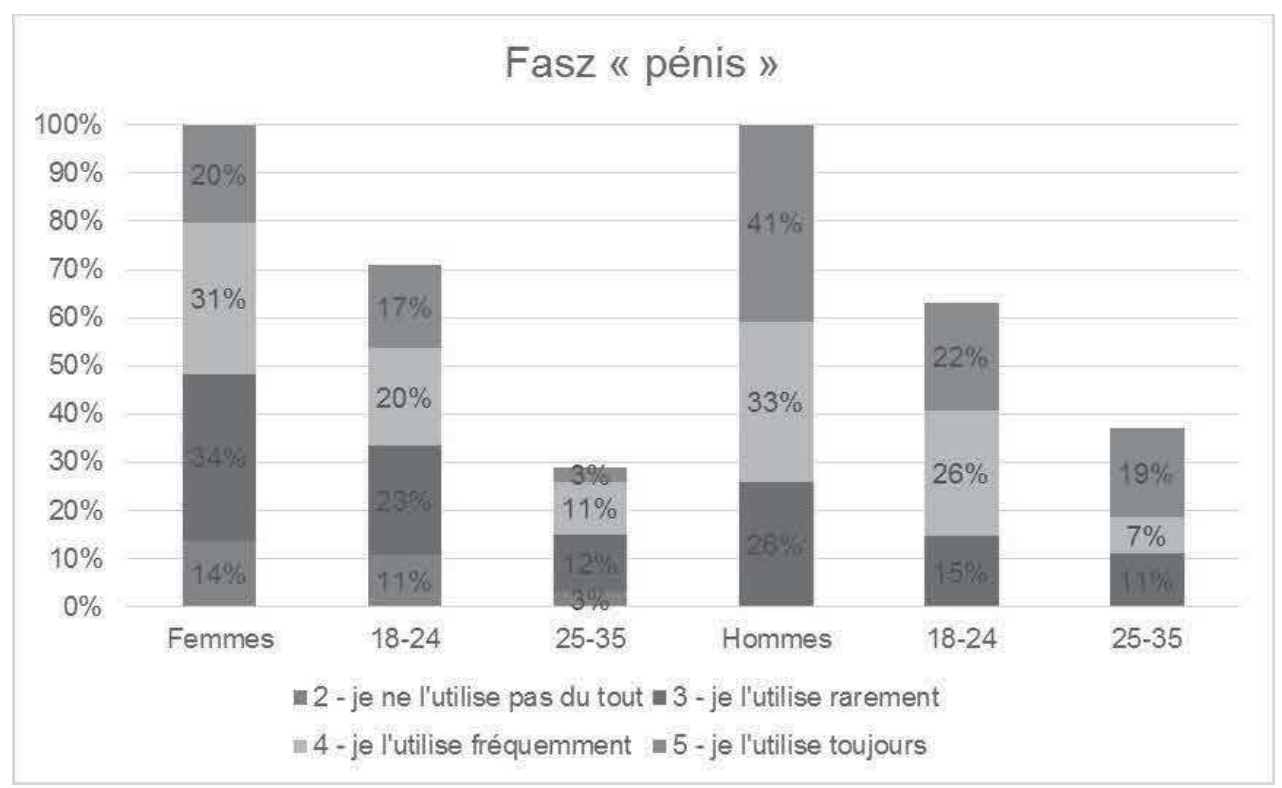

Figure 7 - L'utilisation du mot fasz selon le sexe et l'âge 
Comme la figure 7 en témoigne, le mot fasz «pénis » semble s'inscrire dans la lignée des mots comme picsa «postérieur » et pina « vulve » dans le sens où ce sont les hommes qui l'emploient de façon plus fréquente ${ }^{20}$. Nous constatons, pour les usages constant et fréquent, $74 \%$ pour les hommes contre $51 \%$ pour les femmes. Cette différence apparaît chez les participants âgés de 18 à 24 ans (48\% pour les hommes contre $37 \%$ pour les femmes), mais elle s'avère plus marquante chez ceux âgés de 25 à 35 ans ( $26 \%$ pour les hommes contre $14 \%$ pour les femmes).

Pareillement aux trois mots précédents, une différence s'observe aussi quant à la profession et au lieu de résidence des participants de notre enquête.

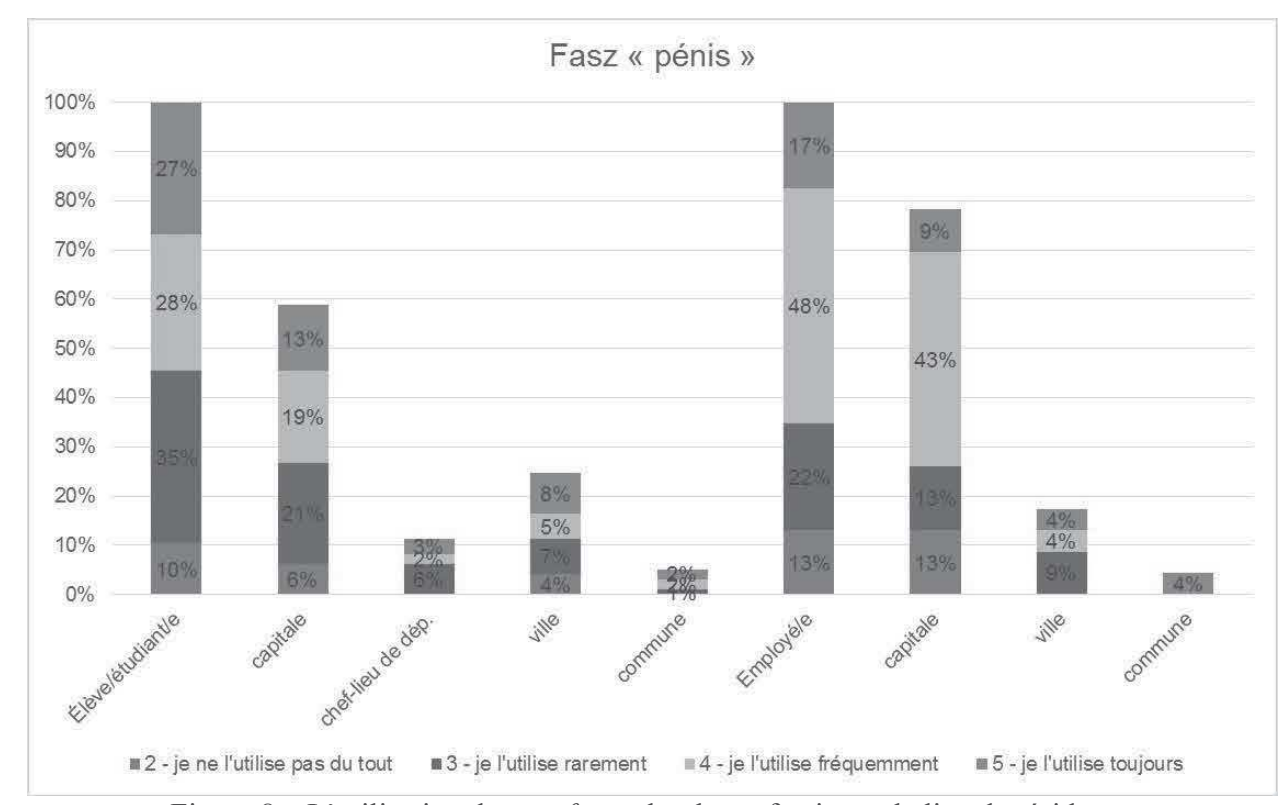

Figure 8 - L'utilisation du mot fasz selon la profession et le lieu de résidence

Les employés déclarent utiliser le mot fasz plus régulièrement que les élèves et étudiants (65\% pour les employés pour les usages constant et fréquent contre $55 \%$ pour les élèves et étudiants pour les mêmes utilisations). Cette différence est particulièrement prononcée chez les habitants de la capitale : $52 \%$ pour les employés contre $32 \%$ pour les élèves et étudiants.

\subsection{D'autres mots actuellement utilisés pour désigner les organes génitaux masculins}

Comme les organes génitaux des femmes, ceux des hommes connaissent également un grand éventail de désignations dans le corpus que nous avons recueilli.

\footnotetext{
${ }^{20}$ Cette affirmation est corroborée par notre travail antérieur concernant le champ lexical d'aimer où nous avons remarqué que l'adjectif fasza «bon, bien», issu du substantif fasz "pénis»par l'adjonction du suffixe $-a$, avait été plus largement utilisé par les hommes. Voir KovÁcs (2021).
}

$$
-54-
$$




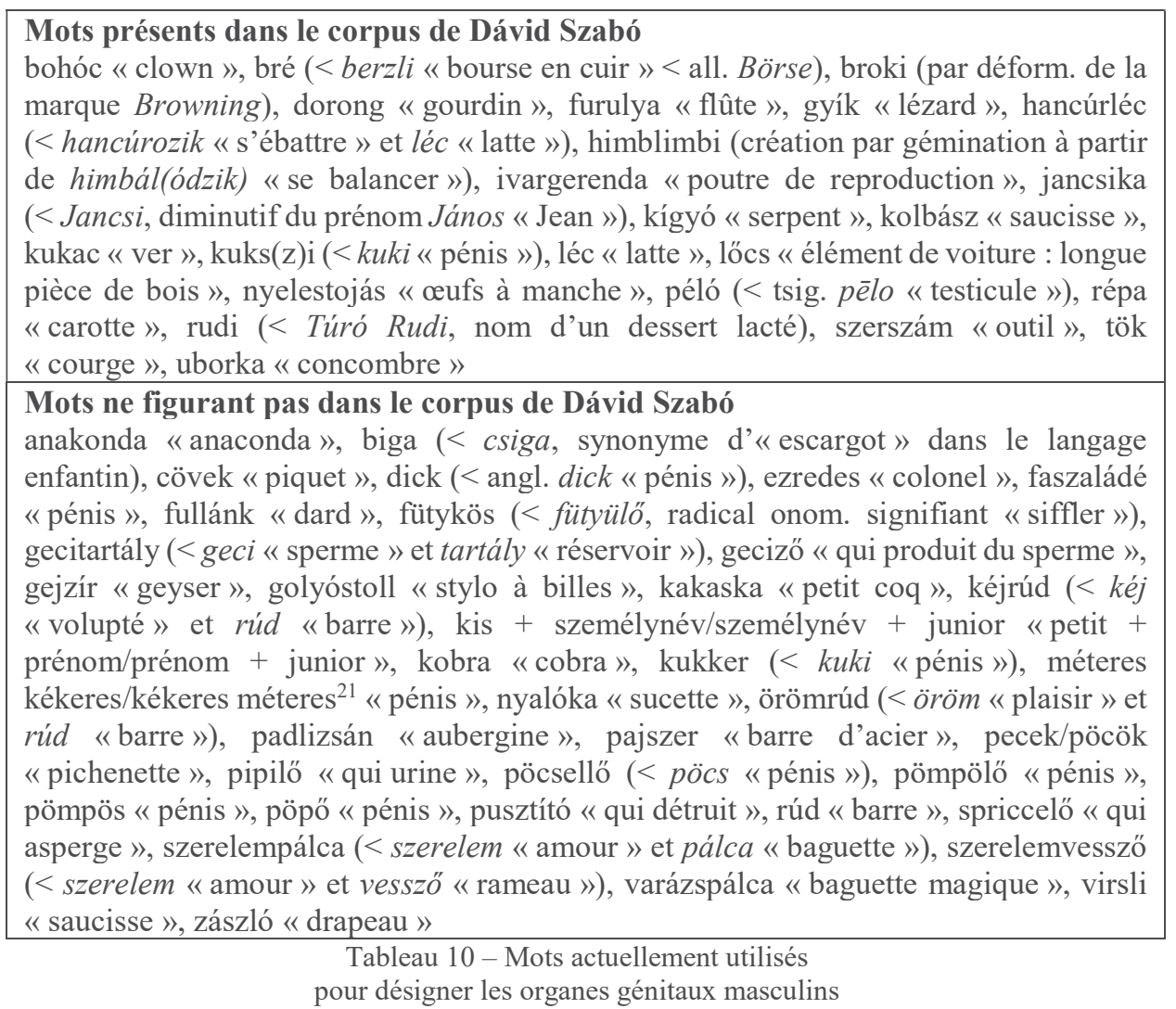

Le tableau ci-dessus montre bien qu'un grand nombre d'exemples mentionnés par les participants de notre enquête sont inclus dans le dictionnaire de Dávid Szabó mais nombreuses sont également les dénominations qui n'y apparaissent pas. Du point de vue des procédés de création lexicale (SZABÓ 2002a, 2004), nos observations ressemblent aux exemples précédents. Kéjrúd < kéj « volupté » + rúd « barre », szerelempálca < szerelem « amour »+ pálca « baguette », etc. présentent la composition; kukker ${ }^{22}<k u k i$ «pénis » met en scène la suffixation précédée de troncation et himblimbi < himbál(ódzik) «se balancer» relève de la création par gémination (processus formels). Les procédés sémantiques sont essentiellement représentés par la métaphore : gyík « lézard », virsli « saucisse », zászló « drapeau », etc. Enfin, comme dans le cas de chaque partie du corps évoquée, nous trouvons également des emprunts tels que, par exemple, dick< angl. dick « pénis » ou péló < tsig. pélo « testicule». Pour terminer, il nous semble important d'attirer l'attention sur une création lexicale. Si le corpus de Dávid Szabó contient le mot jancsika < Jancsi, diminutif du prénom János « Jean » pour renvoyer aux organes génitaux masculins, nous avons remarqué une généralisation de ce phénomène par les

${ }^{21}$ Nous retrouvons également méteres kékeres avec le même sens dans l'argot militaire hongrois (KIS, $2008: 138)$.

${ }^{22}$ Kukker figure dans le corpus de Dávid Szabó mais avec le sens de « lunettes » (SZABÓ, 2014 : 286). 
expressions : kis + személynév/személynév + junior "petit + prénom/prénom + junior ».

\section{Conclusion}

L'objectif principal de notre article était d'examiner, en partant de cinq notions (la bouche, les jambes, le postérieur, les organes génitaux féminins et masculins), l'évolution du champ lexical des parties du corps dans l'argot commun des jeunes Hongrois plus de vingt ans après l'enquête menée par Dávid Szabó. À l'instar de ce que nous avons constaté précédemment dans le cas du champ lexical d'aimer (KOVÁCS, 2021), celui des parties du corps témoigne également du caractère à la fois changeant et immuable de l'argot commun parlé par les jeunes Hongrois. Comme nous l'avons remarqué, les mots en usage au début des années 2000 ne sont utilisés que partiellement par les locuteurs en 2021 et, par conséquent, les jeunes Hongrois emploient d'autres (parfois de nouveaux) mots pour dénommer les différentes parties du corps. D'autres tendances telles que, par exemple, le nombre accru des emprunts à l'anglais peuvent également être observées. Mais quant aux procédés de création lexicale (formels ou sémantiques), ils s'avèrent les mêmes que ceux décrits par SZABÓ (2002a, 2004). Par conséquent, nous arrivons à la même conclusion que dans un travail antérieur (KovÁCS, 2021): si les mots en usage changent, les mécanismes qui gouvernent la création du vocabulaire d'argot commun hongrois, eux, semblent rester immuables.

\section{BIBLIOGRAPHIE}

CAlvet Louis-Jean (2011), La sociolinguistique, Paris, Presses Universitaires de France.

FRANÇOIS Denise (1975), La littérature en argot et l'argot dans la littérature, Communication et langages 27, p. 5-27.

Kis Tamás (1997), Szempontok és adalékok a magyar szleng kutatásához, in : Kis Tamás (dir.), A szlengkutatás útjai és lehetőségei, Debrecen, Kossuth Egyetemi Kiadó, p. 237-293.

Kis Tamás (2008), A magyar katonai szleng szótára, Debrecen, Kossuth Egyetemi Kiadó.

KovÁCs Máté (2021), Le champ lexical d'aimer dans l'argot commun des jeunes Hongrois, Acta Universitatis Lodziensis. Folia Litteraria Romanica 16, p. 105117.

SZABÓ Dávid (2002a), Les procédés de la formation du vocabulaire de l'argot hongrois, Cahiers d'Études Hongroises 10, p. 161-169.

SzABÓ Dávid (2002b), La place de l'emprunt dans l'argot de Budapest, La Linguistique 38, p. 113-127.

SZABÓ Dávid (2004), L'argot des étudiants budapestois, Paris, L'Harmattan.

SZABÓ Dávid (2009), Mots d'homme, mots de femme. À la recherche de différences génolectales en argot hongrois, in: KACPRZAK Alicja, GOUDAILLIER Jean- 
Pierre (dir.), Standard et périphéries de la langue, Łódź, Łask, Oficyna Wydawnicza LEKSEM, p. 313-322. 